\title{
Editorial
}

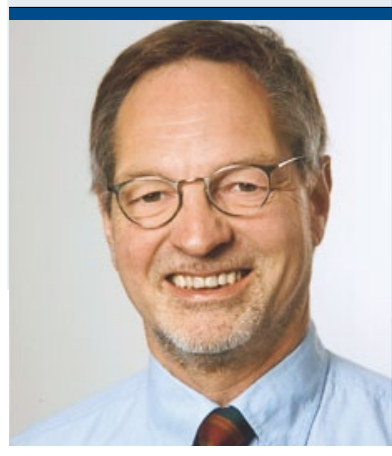

Die niedergelassenen Kollegen beklagen sich über

die DGU-Vollversammlung und bleiben ihr fern,

was den von ihnen kritisierten Stil wiederum begünstigt."

Dr. med. Walther Grohmann,

Chefredakteur und niedergelassener Urologe, München

\section{Die Katze beißt sich in den Schwanz}

A n dieser vordersten Stelle des Heftes möchten wir Ihnen als Leser ganz herzlich danken: In der „Beliebtheitskala“ der unabhängigen LAMed-Facharztstudie* haben Sie als niedergelassene Urologen URO-NEWS wieder in die Spitzengruppe der urologischen Fachzeitschriften gewählt: URONEWS liegt mit einer Reichweite der Leser pro Ausgabe von 74,8\% nach dem „Standesorgan“ Der Urologe $(80,3 \%)$ an zweiter Stelle mit einem Abstand von über $9 \%$ vor den ferner bewerteten urologischen Fachzeitschriften. Dass URO-NEWS somit seit über zehn Jahren in ununterbrochener Reihenfolge in dieser Umfrage einen der Spitzenplätze belegt, erachten wir keineswegs als selbstverständlich. Wir wissen, dass wir dies auch den unermüdlichen Anregungen und der konstruktiven Kritik unserer Leser zu verdanken haben. Unser Dank gilt an dieser Stelle auch allen unseren Autoren sowie dem wissenschaftlichen Beirat von URO-NEWS.

\section{Volles Haus beim BDU, Leere bei der DGU}

Wenn dieses Heft in den Druck geht, wird in Leipzig der Kongress der deutschen Gesellschaft für Urologie (DGU) veranstaltet. Im Rahmen des Kongresses finden die alljährlichen Mitgliederversammlungen der DGU sowie des Berufsverbandes der Urologen (BDU) statt. Analog zur LA-Med-Umfrage läge eine Einschätzung der Attraktivität dieser Fachgesellschaften bei den niedergelassenen Urologen nahe. Ein Maßstab hierfür könnte die Beteiligung der niedergelassenen Urologen an den jeweiligen Vollversammlungen sein. Und da liegen Welten zwischen beiden: Beim BDU ist der Saal in der Regel übervoll - die DGU-Veranstaltung stößt bei den Niedergelassenen dagegen auf mangelnde Resonanz. Es ist zwar verständlich, dass die Berufspolitik, für die der BDU zu zeichnen hat, bei der Basis auf das größere Interesse stößt, zumal es in diesem Jahr um die finanzielle berufliche Existenz gehen wird (siehe dazu den Artikel auf Seite 48). Diesmal stehen aber auch Versäumnisse auf der Tagesordnung, die mit der 2004 unter Federführung der DGU erneuerten Weiterbildungsordnung für Urologen in manchen Bundesländern wie eine Zeitbombe zum Tragen kommen. Stichwort Onkologievereinbarung, bei der auch ein hilfloses Zurückrudern mit einem Statement der Fachgesellschaften bisher so gut wie nichts bewirkt hat. Hinzu kommt jetzt wohl auch noch die Auswirkung auf eine Laborreform bei den Urologen. Aber immerhin: Die Basisdemokratie hat in den letzten Jahren beim BDU funktioniert, was sich bei den letzten Wahlen der jeweiligen Präsidenten widergespiegelt hat.

\section{Alle Jahre wieder}

Zur DGU meinte hingegen ein Kollege, unter Beifall weiterer Anwesender: „Den Bericht zur DGU-Vollversammlung könnt Ihr schon im Heft davor veröffentlichen“, wobei der folgende Text sinngemäß vorgeschlagen wurde: „Nach dem üblichen Weihrauch zum Tätigkeitsbericht und einem kurzen Blick auf die Finanzen werden der vorbestimmte und künftige Präsident wie der weitere Vorstand durchgewinkt und zum Weiteren lässt sich mit Karl Valentin sagen: ,Die (urologische) Zukunft ist auch nicht mehr das, was sie einmal war."

Offensichtlich beißt sich hier die Katze in den Schwanz: Die niedergelassenen Kollegen beklagen sich über die DGU-Vollversammlung und bleiben ihr fern, was den von ihnen kritisierten Stil wiederum begünstigt. Der Schluss, wie das zu ändern wäre, ist leicht zu ziehen.

Ihr
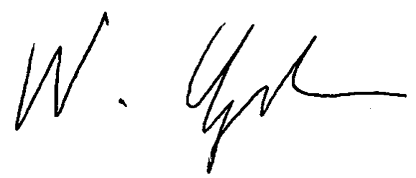

* LA-Med-Facharztstudie 2012,

niedergelassene Urologen, LPA in \% 